\title{
Monte Carlo Study on Induced Antiferromagnetic Phase in Bilayer Films Using Anisotropic Extended Heisenberg Model
}

\author{
E. BÎRsan ${ }^{a, *}$, R. Chis $^{b}$ And A. Dobrita ${ }^{b}$ \\ ${ }^{a}$ Physics Department, "Lucian Blaga" University \\ Dr I. Ratiu Street, No. 5-7, 550024, Sibiu, Romania \\ ${ }^{b}$ Faculty of Engineering "Hermann Oberth", "Lucian Blaga" University \\ Sibiu, Romania
}

(Received March 11, 2008)

\begin{abstract}
In this paper we study the magnetic bearing of a bilayer film in anisotropic extended Heisenberg model, using the Monte Carlo method. In this way, by computing the out-of-plane uniform and staggered magnetization, in-plane uniform magnetization, out-of-plane and in-plane magnetic susceptibility, we point out the existence of an antiferromagnetic phase induced in the superior layer as a consequence of the fixed ferromagnetic arrangement of the spins in the base layer. We can also distinguish the existence of two phase transitions which occur in the physical system: antiferromagnetism $\rightarrow$ mixed phase transition and antiferromagnetism $\rightarrow X Y$-like phase transition, corresponding to the two physical parameter settings.
\end{abstract}

PACS numbers: 75.10.Hk, 75.70.Ak, 75.40.Mg

\section{Introduction}

The development of nanotechnologies during the last years based on the intensive study of physical phenomena which appear in the nanostructured systems, at a theoretical [1-3] level but also experimentally [4-6], draws at this time a growing interest from the research groups of this field. In this research spectrum the thin film study occupies a primordial (essential) place, because of the applications in the hardware industry $[7,8]$, especially for the purpose of obtaining magnetic materials with high data storage capabilities. Surface and interface phenomena

*corresponding author; e-mail: beneugen10@yahoo.com 
represent a great challenge for the present research in the field of thin films. In this work we try to determine the magnetic behavior of a double layered film, that has a base layer (indexed " 0 ") characterized by a fixed antiferromagnetic arrangement of the spins and a superior layer (indexed " 1 "), which is influenced by the base layer, because of the interaction between the magnetic spins. In this approach, we use the Monte Carlo simulation technique [9-11], in Metropolis dynamics, with the purpose of determining the physical sizes of interest, which should emphasize the upper mentioned influence. In this way, we will point out three different phases from the magnetic arrangement point of view: the mixed phase, the antiferromagnetic phase and the $X Y$ phase (plane arrangement of the spins).

\section{Physical model and numerical algorithm}

We consider a thin double layered film, where the two layers have different magnetic properties, characterized by a Heisenberg Hamiltonian, with three types of interactions between the lattice spins: direct exchange interaction, anisotropic exchange interaction, and dipolar interaction. We want to investigate the magnetic behavior of the superior layer under the following condition: the base layer spins have a fixed antiferromagnetic arrangement. The physical system considered (the double layered film) is characterized by a Hamiltonian given by the expression

$$
H=-\sum_{\langle i, j\rangle} J_{i j} \boldsymbol{S}_{i} \cdot \boldsymbol{S}_{j}-\sum_{\langle i, j\rangle} A_{i j} S_{i z} S_{j z}+\sum_{i, j} D_{i j} T_{i j}
$$

The first term describes the exchange interaction between adjacent magnetic spins, the second term is determined by the anisotropic interaction and the third term is represented by the relation

$$
T_{i j}=\frac{\boldsymbol{S}_{i} \cdot \boldsymbol{S}_{j}}{r_{i j}^{3}}-3 \frac{\left(\boldsymbol{S}_{i} \cdot \boldsymbol{r}_{i j}\right)\left(S_{j} \cdot \boldsymbol{r}_{i j}\right)}{r_{i j}^{5}},
$$

which forms the dipolar interaction term between the spins. The mean of the physical sizes implied is the following: $J_{i j}$ represents the direct exchange interaction constant, $A_{i j}$ is the easy-axis anisotropy interaction parameter, and $D_{i j}$ is the dipolar interaction between spins parameter (distance interaction). All sizes in the following equations are being indexed according to the layer which the specified spins are part of, the size of the spins in the two layers being $S_{0}=S_{1}=S=1$. We also assume for the general parameters setting, that remain unchanged: $-J_{00}=J_{11}=J=1, A_{00}=1, D_{00}=0.150$ (the rest of parameters are variable and will be defined in the following sections). In our study we are using Monte Carlo (MC) simulations (Metropolis algorithm, with open boundary conditions (OBC)) in order to obtain the physical quantities of interest: out-of-plane uniform and staggered magnetization, out-of-plane and in-plane magnetic susceptibility, with the aid of which we determined the base layer (" 0 ") influence, characterized by a fixed antiferromagnetic arrangement of the spins, over the superior layer of the film, for which we assume a direct exchange interaction of ferromagnetic type (positive). We intend this way to investigate the magnetic 
behavior of the physical system above defined, characterized by the extended represented Heisenberg Hamiltonian and to find interesting phase transitions, that can appear in the system at the same time with temperature variation. Typically, $10^{3}-10^{4} \mathrm{MC}$ steps per spin are used for balance and $10^{4}$ steps for spin are used for calculating the interesting physical average quantities. In order to investigate the physical properties of the system, we need the statistical average of the physical studied quantities. We use for our simulations the out-of-plane $(O Z)$ and in-plane $(X O Y)$ magnetic susceptibility, given by the following expressions (where \langle\rangle denote the statistical average, which is calculated by taking into account the intermediate states over the Monte Carlo steps and $N=N_{x} N_{y} N_{z}$ means the number of sites of the lattice):

$$
\begin{aligned}
& \chi_{z}=N \frac{\left\langle m_{z}^{2}\right\rangle-\left\langle m_{z}\right\rangle^{2}}{k_{\mathrm{B}} T}, \\
& \chi_{x y}=N \frac{\left\langle m_{x y}^{2}\right\rangle-\left\langle m_{x y}\right\rangle^{2}}{k_{\mathrm{B}} T},
\end{aligned}
$$

here $m_{z}, m_{x y}$ being the $O Z$ and $X O Y$ components of the magnetization vector, $T$ being the absolute temperature (which is measured in $J S^{2} / k_{\mathrm{B}}$ units) and we set $k_{\mathrm{B}}=1$ for the Boltzmann constant.

$$
\boldsymbol{m}=\frac{\sum_{i=1}^{N} \boldsymbol{S}_{i}}{N}
$$

$\boldsymbol{S}_{i}$ meaning the spin at the site " $i$ ". The components of the magnetization are given by the following expressions:

$$
\begin{aligned}
& m_{k}=\frac{\sum_{i=1}^{N} S_{i k}}{N}, \quad k=x, y, z, \\
& m_{x y}=\sqrt{m_{x}^{2}+m_{y}^{2}} .
\end{aligned}
$$

In order to detect the potential antiferromagnetic ordering we also define the $O Z$ component of the staggered magnetization, using the expression

$$
m_{\mathrm{s} z}=\frac{\sum_{n}(-1)^{i+j} S_{z n}}{N} .
$$

\section{The simulation results}

In this section we present the results of our numerical simulation for the physical quantities of interest, for two settings of the parameters specific to the above presented model, which point out the existence of two phase transitions: antiferromagnetism — mixed phase transition and antiferromagnetism $X Y$-like ordering phase transition.

\subsection{Antiferromagnetism - mixed phase transition}

In this paragraph we examine the antiferromagnetism $\rightarrow$ mixed phase transition, in the physical conditions determined by the following physical parameters: 
$A_{01}=A_{11}=1.2, D_{01}=D_{11}=0.150, N_{x}=N_{y}=60$ and $J_{01}=1.0 ; 1.1 ; 1.2$, respectively.

In Fig. 1a and b we present the out-of-plane uniform and staggered magnetization, respectively, where an antiferromagnetic system's behavior in the domain of small temperatures can be observed, while once with the rise in temperature the system moves towards a mixed phase (somewhere in the temperature interval: $T=0.53-0.56)$ where ferromagnetic order zones alternate with magnetic disarray zones of the spins. For the magnetization, taking into account the limited character of the dimension of the film, we can associate the critical temperature with the inflexion point of the graphic representation. The magnetic behavior previously presented is confirmed by Fig. 2a and b, where we present the out-of-plane and inplane magnetic susceptibility, depending on the temperature, respectively. We can observe a slight drop in the critical temperature (associated with the maximum of the magnetic susceptibility) along with the interlayer direct exchange parameter $\left(J_{01}\right)$ decreasing.
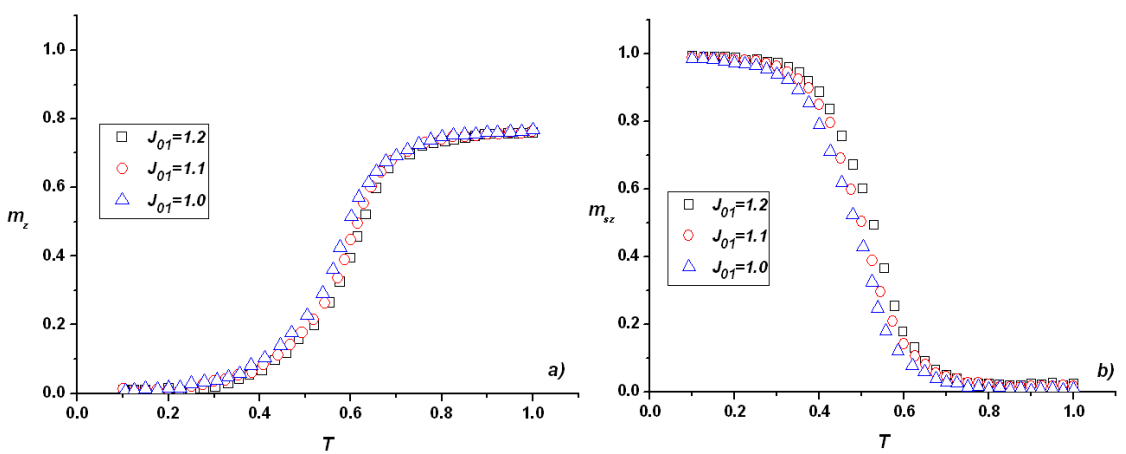

Fig. 1. Out-of-plane uniform (a) and staggered (b) magnetization for different $J_{01}$ values.

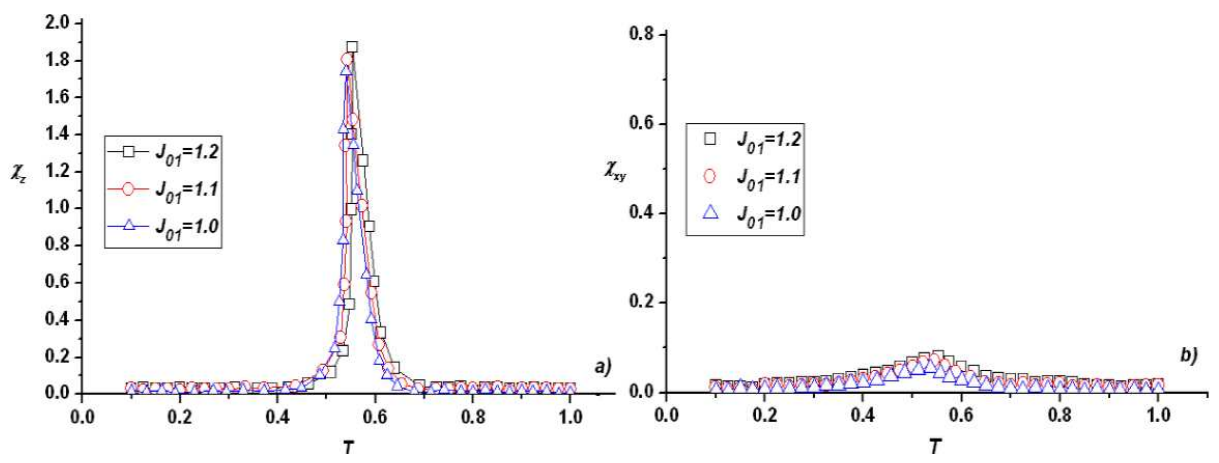

Fig. 2. Out-of-plane (a) and in-plane (b) magnetic susceptibility for different $J_{01}$ values. 


\subsection{Antiferromagnetism - XY-like ordering phase transition}

In this paragraph we want to point out the antiferromagnetic spins ordering $\rightarrow X Y$-like ordering phase transition (triangles in Fig. 2), which is possible to occur when the physical parameters are set like the following: $A_{01}=A_{11}=1.2$, $D_{01}=D_{11}=0.200, N_{x}=N_{y}=60$ and $J_{01}=1.0 ; 1.1 ; 1.2$, respectively.

Figures 3 and 4 present the out-of-plane uniform and staggered magnetization, respectively, as a function of temperature.

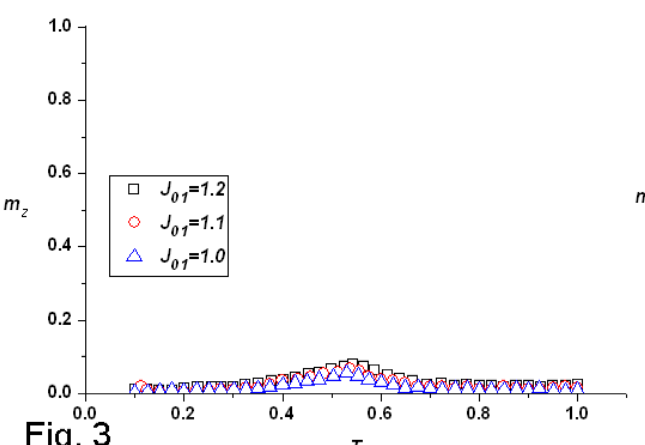

Fig. 3

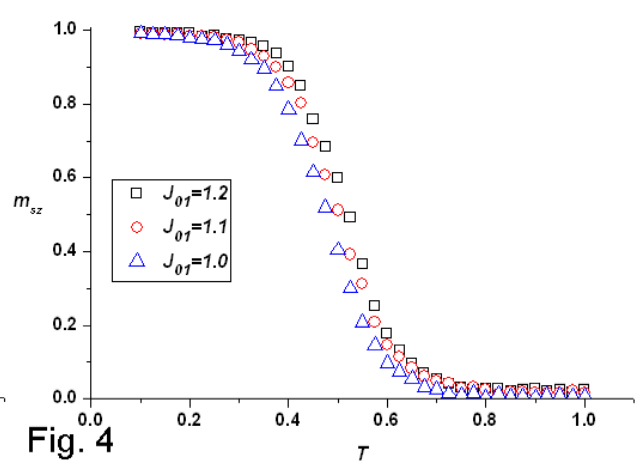

Fig. 3. Out-of-plane uniform magnetization for different $J_{01}$ values.

Fig. 4. Out-of-plane staggered magnetization for different $J_{01}$ values.

Figure 5 shows the in-plane magnetization computed for the physical quantities corresponding to this section (presented above).
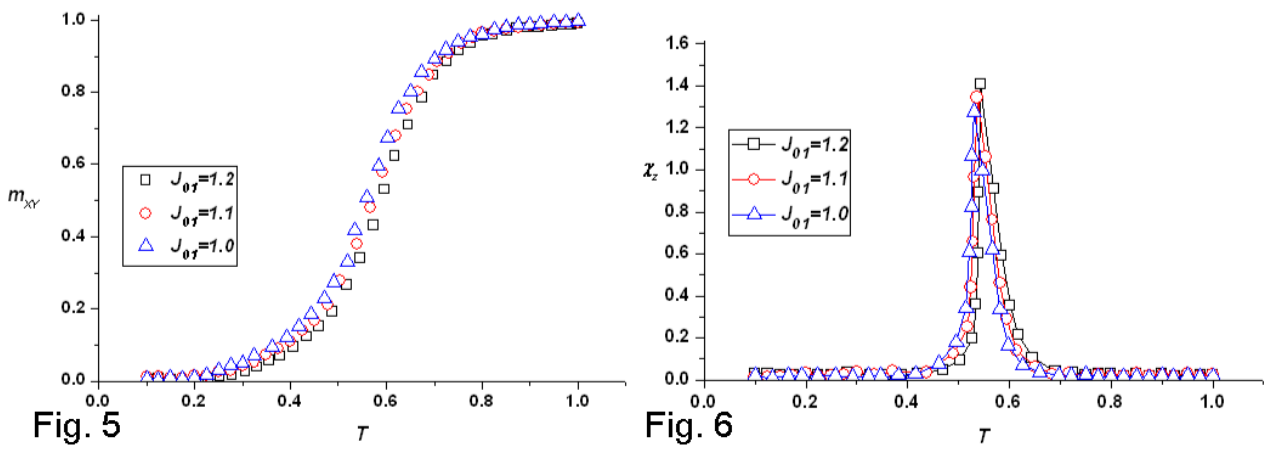

Fig. 5. In-plane uniform magnetization for different $J_{01}$ values.

Fig. 6. Out-of-plane magnetic susceptibility for different $J_{01}$ values.

These results suggest the existence of an antiferromagnetic order of spins in the superior layer in the domain of low temperatures and an in-plane reorientation of these once the temperature rises, this phenomenon being reported experimentally $[12]$. 
The conclusions mentioned above are strengthened by the out-of-plane and in-plane magnetic susceptibilities graphics (Figs. 6 and 7, respectively). The physical system presented in this study, characterized by the extended Heisenberg Hamiltonian (which includes both the anisotropic and dipolar interaction) can be described from a magnetic point of view by a phase diagram like the one presented in Fig. 8 (qualitative aspect).

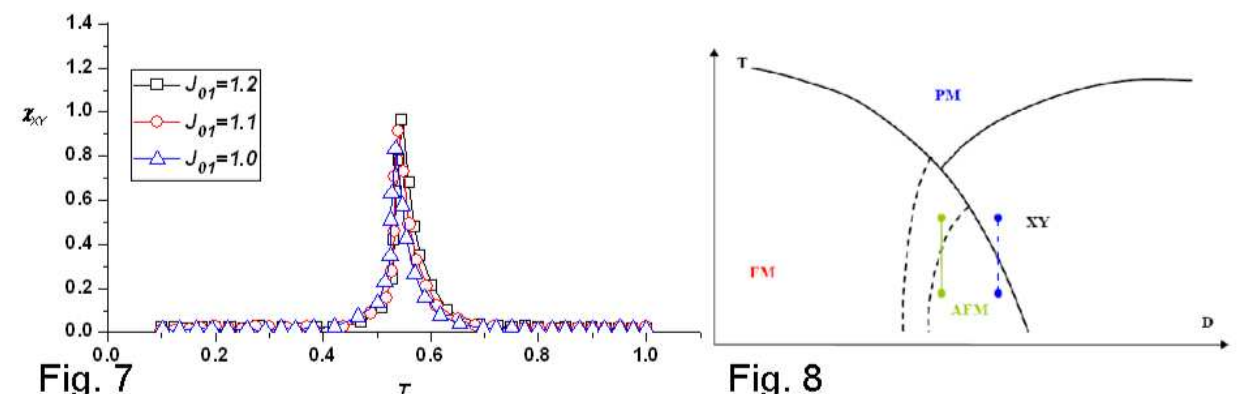

Fig. 7. In-plane magnetic susceptibility for different $J_{01}$ values.

Fig. 8. The phase diagram (qualitative aspect).

Thus we relieve two phase transitions, which can occur in the system: antiferromagnetism $\rightarrow$ mixed phase transition (the green segment in Fig. 8), the mixed phase being placed in the region between the dotted lines of the phase diagram (see Fig. 8) and antiferromagnetism $\rightarrow X Y$-like phase transition (the right dashed segment in Fig. 8), respectively. We emphasize in this way the appearance of the antiferromagnetic arrangement in the superior layer as a consequence of its interaction with the base layer, given the anisotropic discontinuities of the parameters, which occur at the interface of the two layers.

\section{Conclusions}

The magnetism of nanostructured materials represents an important field in the research spectrum at the present day, because of the interesting physical phenomena which occur as a consequence of the dimension of the systems and the physical interactions which describes them. This way in this paper we pointed out the appearance of an antiferromagnetic phase induced in the superior layer of a double layered thin film, because of the antiferromagnetic arrangement in the base layer. We pointed out also the existence of two phase transitions, according to the spin magnetic arrangement states in the investigated layer: antiferromagnetism $\rightarrow$ mixed phase transition (the green segment in Fig. 8) and antiferromagnetism $\rightarrow X Y$-like phase transition (the blue segment in Fig. 8). We observe that the interlayer direct exchange interaction enhancement favors the stability of the induced antiferromagnetic phase and leads to a slight critical temperature rising. 


\section{References}

[1] M. Pavkov, M. Škrinjar, D. Kapor, S. Stojanović, Physica A 280, 465 (1999).

[2] H. Jang, M. Grimson, Phys. Rev. E 63, 066119 (2001).

[3] H.W. Diehl, Int. J. Mod. Phys. B 11, 3503 (1997).

[4] J. van Driel, F.R. de Boer, K.-M.H. Lenssen, R. Coehoorn, J. Appl. Phys. 88, 975 (2000).

[5] P.J. van der Zaag, R.M. Wolf, Phys. Rev. Lett. 80, 608 (1998).

[6] T.J. Moran, J. Nogués, D. Lederman, I.K. Schuller, Appl. Phys. Lett. 72, 617 (1998).

[7] A. Zangwill, Physics at Surfaces, Cambridge University Press, Cambrige 1988.

[8] P.M. Lévy, Solid State Phys. 47, 367 (1994).

[9] J.M. Thijssen, Computational Physics, Cambridge University Press, Cambridge 1999.

[10] S.E. Koonin, D.C. Meredith, Computational Physics, Addisson-Wesley, Reading (MA), 1990.

[11] D.P. Landau, K. Binder, A Guide to Monte Carlo Simulations in Statistical Physics, Cambridge University Press, Cambridge 2002.

[12] R. Allenspach, A. Bischof, Phys. Rev. Lett. 69, 3385 (1992). 